\title{
OTIOTOMICS
}

Revista de economía, empresa y sociedad

Dossier «Prevención de riesgos laborales: tendencias en tiempo de crisis»

UNA HERRAMIENTA PARA REDUCIR RIESGOS

\section{La gestión de la seguridad vial según la Norma ISO 39001}

\section{Agustín Sánchez-Toledo Ledesma}

Sánchez-Toledo \& Asociados

\section{Xavier Baraza Sánchez}

Profesor de la Universitat Oberta de Catalunya (UOC). Estudios de Economía y Empresa

RESUMEN La seguridad vial (road traffic safety -RTS-) es una preocupación global. Se estima que cada año, en las vías públicas del mundo, alrededor de 1,3 millones de personas fallecen y entre 20 y 50 millones sufren lesiones, y que esta cifra aumenta. En este sentido, la Norma ISO 39001, que se analiza en el presente artículo, suministra una herramienta que permite ayudar a las organizaciones a reducir, y en última instancia eliminar, la incidencia y riesgo de las muertes y heridas graves derivadas de los accidentes de tráfico.

PALABRAS CLAVE seguridad vial; ISO 39001; gestión; accidente en carretera

\section{Road Traffic Safety management by ISO 39001}

\begin{abstract}
Road traffic safety is a global concern. It is estimated that each year in the public roads worldwide, over 1.3 millions of people die, between 20 and 50 million suffer some injuries and the number keeps rising. In this sense, the regulation ISO 39001 which is analyzed in this article provides a tool for the organizations not only to reduce but even to eliminate the impact and risk of death and serious injuries as consequences of traffic accidents.
\end{abstract}

KEYWORDS road traffic safety; ISO 39001; management; road accident

\section{Introducción}

Se define en la Norma ISO 39001 la seguridad vial (road traffic safety -RTS-) como los «factores y condicionantes de los accidentes de tráfico y otros incidentes de tráfico que tienen un impacto, o potencial de tenerlo, en la muerte o heridas graves de los usuarios de la vía».

La seguridad vial es una preocupación global. Los datos del Informe sobre la situación mundial de la seguridad vial 2013, publicado por la Organización Mundial de la Salud, pronostican que para 2030 los accidentes de tráfico 
podrían ser la quinta causa de mortalidad si no se toman medidas. En la actualidad se estima en 1,3 millones los fallecidos por año en los accidentes de tráfico, lo que los convierte en la octava causa de mortalidad a nivel mundial y la primera entre los jóvenes de 15 a 29 años. El impacto socioeconómico y en la salud es, por lo tanto, muy importante y merece una especial atención.

La Norma ISO 39001 aparece como respuesta a la necesidad de disponer de herramientas que permitan mejorar la gestión de la seguridad vial. Dicha norma se crea en el seno el Comité Técnico de Normalización internacional ISO/TC 241 «Road-Traffic Safety Management Systems», encargado del desarrollo de la Norma ISO 39001:2012 «Road traffic safety management systems - Requirements with guidance for use», en el seno de la Organización Internacional de Normalización (ISO). Esta norma se publicó en octubre de 2012.

En el ámbito nacional, el seguimiento de dicha norma se realizó por parte del Comité Técnico de Normalización AEN/CTN 199 «Equipamiento para la gestión del tráfico», cuya Presidencia desempeña la Dirección General de Tráfico (DGT). Finalmente, la publicación a nivel nacional como norma UNE «UNE-ISO 39001:2013 Sistemas de gestión de la seguridad vial. Requisitos y recomendaciones de buenas prácticas» se realizó el 10 de abril del año 2013, por parte de AENOR, como entidad legalmente responsable del desarrollo de las normas técnicas en España.

La ISO 39001 busca ser una herramienta flexible que ayude a las organizaciones públicas y privadas a reducir, y en última instancia eliminar, los fallecimientos y las lesiones graves consecuencia de los accidentes de tráfico. Para alcanzarlo, las organizaciones deben centrarse en mejorar su desempeño, que en este contexto hace referencia a los resultados medibles de su gestión que contribuyen a la seguridad vial, centrándose en cuestiones como son el factor humano, los vehículos, las carreteras y los aspectos organizativos y de respuesta ante emergencias.

El sistema de gestión definido en la norma ISO 39001 se centra en la organización y en sus objetivos y metas en materia de seguridad vial, pero con un enfoque holístico, identificando, consultando y estableciendo comunicación con las partes interesadas. La norma proporciona orientación sobre la planificación de actividades para alcanzar dichos objetivos y metas mediante un enfoque de sistema seguro. La norma promueve también el uso de procesos iterativos tipo PDCA (planificar, hacer, verificar, actuar), base de la mejora continua. El sistema de gestión se enfoca en los resultados y basa sus intervenciones en evidencias.

La norma no pretende especificar requisitos técnicos o de calidad específicos de los productos y servicios de transporte (por ejemplo, vías públicas, señales de tráfico y semáforos, automóviles, tranvías, servicios de transporte de bienes y personas, servicios de rescate y emergencia), ni una uniformidad en la estructura o la documentación del sistema de gestión; los requisitos dados en la norma deben entenderse como genéricos y adaptables a cada organización. No se busca especificar requisitos técnicos y de calidad.

\section{Objeto y campo de aplicación de la Norma ISO 39001}

La ISO 39001 especifica los requisitos para un sistema de gestión de la seguridad vial (road traffic safety -RTS-) que permita a una organización que interactúa con el sistema vial reducir las muertes y heridas graves derivadas de los accidentes de tráfico. Los requisitos de la ISO 39001 incluyen el desarrollo y la aplicación de una política de RTS adecuada, el desarrollo de los objetivos de RTS y los planes de acción que tengan en cuenta los requisitos legales y de otro tipo de obligaciones que la organización suscriba, así como información sobre los elementos y criterios relacionados con la RTS que la organización identifica como aquellos que puede controlar y modular.

La ISO 39001 es aplicable a todas las organizaciones, sin importar su tipo, tamaño o el servicio que prestan. En este sentido, los objetivos perseguidos son:

- Mejorar el desempeño en la RTS.

- Establecer, implementar, mantener y mejorar un sistema de gestión de la RTS.

- Asegurar su propia conformidad con sus políticas RTS.

- Demostrar su conformidad con esta norma internacional. 
La RTS es una responsabilidad compartida. La ISO 39001 no invalida la obligación que tienen los usuarios de la vía de respetar la ley y actuar con responsabilidad. Su propósito es ayudar a la organización a fomentar el respeto a la ley de los usuarios y ha de servir, en todo caso, como complemento a la ley.

A la hora de implantar el sistema de gestión de la seguridad vial, lo primero que debe hacer la organización es analizar su papel en el sistema vial y conocer cómo impactan sus actividades, sus servicios, sus empleados, etc., sobre la seguridad vial.

\section{Estructura de la Norma ISO 39001}

La estructura de la ISO 39001 es similar a la de otro tipo de normas ISO, por lo que la gestión de la seguridad vial puede integrarse en, o hacerse compatible con, otros sistemas de gestión y procesos de la organización. La estructura es:
0. Introducción
1. Objeto y campo de aplicación
2. Normas para consulta
3. Términos y definiciones
4. Contexto de la organización
5. Liderazgo
6. Planificación
7. Soporte
8. Operación
9. Evaluación del desempeño
10. Mejora

La norma se complementa con tres anexos que se detallan a continuación:
A. Guía de uso de la Norma ISO 39001.
B. Trabajos internacionales relacionados con los sistemas de gestión de la seguridad vial.
C. Correspondencia entre las normas ISO 39001:2012, ISO 9001:2008 e ISO 14001:2004.

Con esta norma no se busca uniformidad en la estructura de la gestión de la RTS o uniformidad en la documentación.

Todos los requisitos de esta norma internacional son genéricos. Cuando un requisito de esta norma internacional no se pueda aplicar debido a la naturaleza de la organización y su producto, puede considerarse para su exclusión si se documentan la propia exclusión y su correspondiente motivación.

\section{Factores de desempeño de la Norma ISO 39001}

En esta norma tienen especial importancia aquellos puntos que hacen referencia a los factores de desempeño, que describen los diferentes elementos de seguridad vial que las organizaciones deben tener en cuenta en sus sistemas de gestión de la seguridad vial.

En este sentido la norma ISO 39001 detalla una lista de factores de exposición al riesgo, factores de resultado de seguridad vial finales y de resultado de seguridad vial intermedios, en función del contexto de la organización y de los riesgos y oportunidades que se hayan identificado. Estos factores son: 
a) Factores de exposición al riesgo: Distancia recorrida y volumen de tráfico, desglosando por tipo de vehículo y usuario de la vía, vengan o no afectados por la organización.

b) Factores finales de resultado de seguridad vial: Por ejemplo, el número de muertos y heridas graves.

c) Factores intermedios de resultado de seguridad vial: Estos factores de desempeño se refieren a la planificación, el diseño y el uso seguros de la red vial y los productos y servicios dentro de esta, las condiciones para la entrada y salida de estos productos, servicios y usuarios, así como la recuperación y rehabilitación de las víctimas de los accidentes de tráfico.

Las organizaciones de diferentes tipo y tamaño que cumplan los requisitos de esta norma internacional documentarán y manejarán un sistema de gestión de la RTS distinto en función de su tamaño, alcance y complejidad, a pesar de que el objetivo de la RTS sea el mismo a largo plazo.

La tabla 1 ilustra los diferentes contextos de RTS para diferentes tipos de organizaciones y los factores de desempeño en RTS que pueden ser más adecuados. La identificación del contexto y los factores de desempeño en RTS es un elemento clave de esta norma internacional. Los siguientes ejemplos pretenden servir de referencia.

Tabla 1. Factores de desempeño para diferentes tipos de organizaciones

\begin{tabular}{|c|c|}
\hline Organización & Factores de desempeño \\
\hline \multirow[t]{2}{*}{$\begin{array}{l}\text { Transporte } \\
\text { de personas y bienes } \\
\text { (una compañía pequeña de taxis) }\end{array}$} & $\begin{array}{l}\text { La base del negocio de transporte de personas tiene un impacto directo en la seguridad vial } \\
\text { de los empleados, clientes y otros usuarios de la vía. Entre las partes interesadas a las que } \\
\text { la compañía de taxis pudiera necesitar consultar se incluyen los clientes (por ejemplo, en lo } \\
\text { relativo al uso de medios de sujeción), los conductores (respecto a la velocidad) y aquellos que } \\
\text { tienen que ver con la compra de vehículos (respecto a la selección de vehículos seguros) y el } \\
\text { mantenimiento (que garantice que se mantiene la seguridad). }\end{array}$ \\
\hline & $\begin{array}{l}\text { Como factores de desempeño clave de RTS de una compañía de taxis, se debería incluir la } \\
\text { incapacidad del conductor (por ejemplo, por fatiga, alcohol o drogas), la velocidad de circula- } \\
\text { ción, los cinturones de seguridad de conductor y pasajero, la elección de vehículos y su man- } \\
\text { tenimiento, y la planificación de viajes. Como herramientas clave se incluyen la conformidad de } \\
\text { los mecanismos de sujeción así como el estado del permiso de conducción. }\end{array}$ \\
\hline \multirow[t]{2}{*}{$\begin{array}{l}\text { Transporte de personas y bienes } \\
\text { (un proveedor de servicios de trans- } \\
\text { porte por carretera) }\end{array}$} & $\begin{array}{l}\text { Los vehículos comerciales están relacionados con una cantidad desmesurada de muertos en } \\
\text { las vías públicas del mundo. Los proveedores de servicios de transporte tienen una respon- } \\
\text { sabilidad en la RTS de sus empleados, terceras partes con los que ellos contactan, así como } \\
\text { en las comunidades en las que realizan su trabajo. Además, tienen responsabilidad con sus } \\
\text { clientes para que las mercancías lleguen a destino en condiciones. }\end{array}$ \\
\hline & $\begin{array}{l}\text { Como factores de desempeño clave de RTS, se incluyen la selección de conductores, y cómo } \\
\text { estos se gestionan y motivan para garantizar las destrezas y comportamientos adecuados, } \\
\text { particularmente en lo que afecta a la gestión de la velocidad y la aptitud del conductor. La } \\
\text { selección y el uso de vehículos más apropiados a su objeto, diseñados y equipados para } \\
\text { reducir el riesgo de accidentes de tráfico, así como el riesgo de muerte y heridas graves de los } \\
\text { ocupantes del vehículo y resto de los usuarios de la vía, y las inspecciones y el mantenimiento } \\
\text { para asegurar su adecuación a la carretera. Se debe gestionar la carga de manera adecuada } \\
\text { de modo que no se produzcan sobrecargas, así como la estiba segura. Una planificación } \\
\text { segura del viaje que garantice la ruta más adecuada, velocidades y horas de conducción. La } \\
\text { consideración de otros usuarios vulnerables de la vía y, en caso de incidente, la preparación } \\
\text { para las emergencias. }\end{array}$ \\
\hline
\end{tabular}




\section{Organización}

\section{Transporte de personas y bienes} (una organización multinacional de ventas y marketing)

\section{Factores de desempeño}

La conducción dentro de una compañía de negocios representa la actividad de mayor riesgo de una empresa que opera con flotas a escala regional o global. Los comerciales, agentes de servicios y resto de los conductores pueden estar entre el 40 y el $60 \%$ de su tiempo conduciendo vehículos de la propia compañía, en régimen de leasing, renting, alquiler u otro tipo. Como tales, las empresas tienen la obligación de asegurar la salud y seguridad vial de sus empleados y de las comunidades en las que trabajan.

Como factores de desempeño clave de RTS, se incluyen el conocimiento de los riesgos de seguridad de su flota, por ejemplo, el riesgo de muerte o heridas; el acceso tanto de conductores como de vehículos al sistema vial; las políticas en materia de velocidad, alcohol, uso del casco y los cinturones de seguridad, la fatiga y distracción del conductor, la selección y el mantenimiento de los vehículos, la planificación de los viajes; la gestión de la RTS de los contratistas o distribuidores; la responsabilidad social corporativa a través de la participación en la defensa de la seguridad vial, y el apoyo a las iniciativas de la comunidad en materia de seguridad vial.

\section{Generador de demanda de tráfico} (una escuela)

\section{Generador de demanda de tráfico} (un supermercado)

\section{Diseño vial}

y operaciones

(un titular de vía)
La RTS no es el negocio esencial de una escuela, pero al día se hacen muchos viajes de ida al colegio por parte de una serie de usuarios vulnerables, en términos tanto de madurez como de exposición. Las heridas derivadas de los accidentes de tráfico son la causa principal de muerte de los niños en edad escolar. Las partes interesadas son el personal, los estudiantes y padres, las autoridades locales de planificación, los titulares de la vía, los operadores de transporte (que pudieran requerir la mejora de los programas de seguridad vial).

Como factores de desempeño en RTS clave de una escuela, se deberían incluir los diferentes modos de ir a la escuela y volver de ella, la planificación segura de estos viajes y el uso apropiado de elementos de seguridad (por ejemplo, cascos, cinturones) y elementos asistentes a la visibilidad (por ejemplo, ropa reflectante, luces).

El negocio principal de transporte de bienes, incluidas las mercancías peligrosas (como los combustibles), el reparto a domicilio de mercancías a los clientes, así como la distribución a los supermercados, pueden tener un importante impacto en la seguridad vial. Un supermercado grande debería considerar estos factores de seguridad vial que afectan a sus empleados y clientes. Como partes interesadas se incluirán las autoridades responsables de la planificación, los titulares de la vía y las empresas de reparto.

Un supermercado grande tiene como consideraciones clave del impacto que puede tener en la seguridad vial de los alrededores todos aquellos factores que pueden contribuir a un entorno seguro de sus clientes, sea en la zona de aparcamiento (que tiene un uso mixto de peatones, niños, vehículos de motor grandes y pequeños), sea en los accesos y salidas.

Hay una serie de factores de desempeño que pueden ser importantes, entre los que se incluye la planificación y diseño seguro, el uso y operación de la infraestructura vial de la que se es responsable, el uso de equipos de seguridad por parte de sus conductores, la velocidad segura y los tipos de vehículos que hacen el reparto.

El negocio principal de la construcción de una red vial tiene un impacto en la seguridad vial de todos los usuarios de la red. Las partes interesadas de un titular de la vía local son los estudios de ingeniería civil y los operadores de servicios (que deberían ser capaces de dar el nivel de seguridad vial que se pide), los principales usuarios (que deben aceptar los requisitos del uso seguro) y las agencias de vigilancia (que tienen que vigilar las normas que afectan a usuarios/ vehículos, así como establecer limitaciones a la seguridad inherente de la red).

Como factores clave del desempeño en RTS, se incluyen todos aquellos que se encuentran dentro de la categoría de la planificación y diseño seguro, y el uso y operación de la red vial. Se incluyen en los anteriores factores los relativos a los sistemas de contención laterales y frontales de los usuarios vulnerables, así como los correspondientes límites de velocidad y su cumplimiento. Las herramientas de control pueden estar integradas en un análisis de gestión de la red de mayor rango, así como en los informes de seguimiento de la vigilancia. 


\section{La mejora del sistema de gestión de la seguridad vial}

Para que el sistema de gestión de la seguridad vial sea efectivo de manera continuada, hace falta que una organización tenga procedimientos que identifiquen las no conformidades, reales o potenciales, y tome acciones correctivas o preventivas que, de forma preferente, eviten los problemas antes de que sucedan.

Ejemplo de problemas que pueden dar lugar a no conformidades, en relación con el desempeño del sistema de gestión de la RTS, incluyen el fallo de la alta dirección en la evidencia del compromiso, en el establecimiento de los objetivos de RTS o en la definición de responsabilidades; en el apoyo y promoción del sistema o en la disposición de recursos suficientes, en la evaluación periódica de la conformidad con el sistema, en el registro de los incidentes de tráfico o la implementación de acciones correctivas a tiempo, y en el mantenimiento de la documentación adecuada. En relación con el desempeño en RTS, como ejemplos se incluyen el fallo al implementar las actividades planificadas, el fallo en alcanzar los objetivos de RTS y metas de RTS, o altas tasas de accidentes de tráfico y otros incidentes que no se han tratado.

\section{Conclusiones}

En ciertos grupos de edad las muertes por accidentes de tráfico son las de mayor número. Si no se hace nada, la situación irá a peor; nos encontramos en un punto de inflexión. La ISO 39001 es una norma flexible y útil para todo tipo de organizaciones que trabajan con el claro objetivo de reducir los accidentes de tráfico.

Los accidentes de tráfico constituyen un problema mundial que debe ser abordado. Tener una norma, como la ISO 39001, ayudará a:

- Salvar vidas y reducir las lesiones.

- Reducir costes.

- Aumentar la seguridad, reducir el impacto ambiental y construir la imagen de marca de la organización.

- Tener un impacto positivo en el resto del sistema de tráfico.

- Fomentar un comportamiento más seguro.

- Apoyar a la industria del automóvil para desarrollar e introducir productos y servicios más seguros.

- Acceso al «bonus» establecido en el Real Decreto 404/2010.

- Reducciones en las primas de seguros.

- Reconocimiento de la administración en concursos relacionados con el transporte y el sistema vial.

\section{Bibliografía}

AGENCIA ANDALUZA DE LA ENERGÍA (2006). Guía práctica para la elaboración e implantación de planes de transporte al centro de trabajo. Madrid: Instituto para la Diversificación y Ahorro de la Energía.

ASOCIACIÓN ESPAÑOLA DE NORMALIZACIÓN (2013). UNE-ISO 39001:2013 Sistemas de gestión de la seguridad vial. Requisitos y recomendaciones de buenas prácticas. Madrid: AENOR.

DIRECCIÓN GENERAL DE TRÁFICO (2011). estrategia de seguridad vial 2011-2020. MADRID: DIRECCIÓN GENERAL DE TRÁFICO.

MARTíNEZ, R.; GONZÁLEZ, J. A.; TOLEDO, F. (2011). Guía para las actuaciones de la Inspección de Trabajo y Seguridad Social en materia de seguridad vial en las empresas. Madrid: Ministerio de Trabajo e Inmigración.

ORGANIZACIÓN MUNDIAL DE LA SALUD (2013). Informe sobre la situación mundial de la seguridad vial. Ginebra: Organización Mundial de la Salud. 
SÁNCHEZ, J. M. (2015). Implantación de sistemas de gestión de la seguridad vial. La norma ISO 39001. Madrid: Fundación Confemetal.

UNIÓN EUROPEA (2010). «Hacia un espacio europeo de seguridad vial: orientaciones políticas sobre seguridad vial 2011-2020». Comunicación de la Comisión al Parlamento Europeo, al Consejo, al Comité Económico y Social Europeo y al Comité de las Regiones, de 20 de julio de 2010.

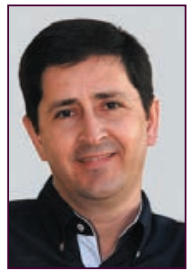

\section{Agustín Sánchez-Toledo Ledesma}

asanchez_toledo@uoc.edu

\section{Sánchez-Toledo \& Asociados}

Ingeniero y doctor en Ingeniería con más de veinte años de experiencia, nacional e internacional, en sistemas de gestión de la seguridad y salud en el trabajo. En la actualidad es director de Sánchez-Toledo \& Asociados y profesor de varias universidades, entre ellas es colaborador docente de la Universitat Oberta de Catalunya. Executive MBA por el IE Business School, máster en Prevención de riesgos laborales (tres especialidades) y máster en Dirección de la calidad y el medio ambiente. Medalla de Oro al Mérito Profesional y Premio Prever. Presidente del Consejo Asesor de la revista Formación de Seguridad Laboral.

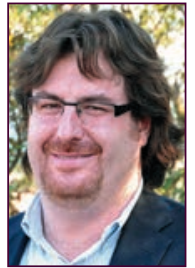

\section{Xavier Baraza Sánchez}

jbaraza@uoc.edu

Profesor de la Universitat Oberta de Catalunya (UOC). Estudios de Economía y Empresa

Ingeniero Químico Industrial y doctor en Ingeniería del Medio Ambiente y del Producto por la Universidad de Barcelona. Máster en Prevención de riesgos laborales (tres especialidades) por la Universidad Politécnica de Cataluña y máster en Dirección y administración de empresas por la Escuela Europea de Negocios. Ha sido director de Seguridad, salud y medio ambiente de una empresa del sector de la química fina entre los años 2005 y 2010. Actualmente es profesor de los Estudios de Economía y Empresa de la Universitat Oberta de Catalunya y director del máster universitario de Prevención de riesgos laborales de esta universidad. Autor de varios libros en materia de PRL y de artículos en revistas de referencia. Miembro del grupo de investigación consolidado DigiBiz (Digital Business Research Group).

Los textos publicados en esta revista están -si no se indica lo contrario- bajo una licencia Reconocimiento-Sin obras derivadas 3.0 España de Creative Commons. Puede copiarlos, distribuirlos y comunicarlos públicamente siempre que cite su autor y la revista y la institución que los publica (autoría, nombre de la revista, institución editora); no haga con ellos obras derivadas. La licencia completa se puede consultar en http://creativecommons.org/licenses/by-nd/3.0/es/deed.es.

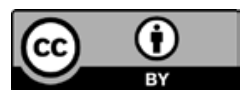

